\title{
Mentalidad localista y hechura de políticas de comercio exterior: lecciones del México subnacional
}

\author{
Parochial Mindset and Foreign Trade Policy-making: \\ Lessons from Subnational Mexico
}

DOI: $10.32870 /$ mycp.v8i24.593

\author{
Antonio Cáñez Cota ${ }^{1}$
}

\begin{abstract}
Resumen
El objetivo del presente artículo es reflexionar sobre la mentalidad localista como un elemento a considerar en la hechura de políticas de comercio exterior subnacionales, para el caso específico del estado de Sonora, México. La estrategia metodológica estuvo basada en entrevistas semiestructuradas a expertos, donde se entrevistaron nueve actores vinculados directamente con la política de comercio exterior, tres del sector público, tres del sector privado y tres del sector universitario. El hallazgo principal indica que la mentalidad localista es un obstáculo para la hechura de políticas subnacionales de comercio exterior efectivas, ya que la poca apertura mentaly comercial afecta negativamente el aprovechamiento de las ventajas internacionales. Dicha mentalidad localista se mantiene y refuerza con la intensa imbricación de la clase empresarial con la clase política, donde no existen los contrapesos necesarios para tomar decisiones en competencia. Entonces, el patrón predominante en las decisiones de política pública responde más a mantener el statu quo local, que a competir en el mercado internacional.
\end{abstract}

Palabras clave: mentalidad localista, políticas subnacionales, comercio exterior, TLCAN, México.

\begin{abstract}
The purpose of this article is to reflect on the parochial mentality as an issue to consider in the policymaking of subnational foreign trade policies, for the specific case of Sonora, Mexico. The methodological strategy was based on semi-structured interviews with experts, where nine actors were interviewed, those directly linked to the foreign trade policy, three from the public sector, three from the private sector, and three from the university sector. The main finding indicates that the parochial mentality is an obstacle to the effective policy-making on the subnational foreign trade arena since the lack of mental and commercial openness negatively affects the exploit of international opportunities. This parochial mentality is maintained and reinforced by the intense imbrications of business groups with political groups, where there are not the necessary check and balances to make competing decisions. Thus, the predominant policy-making pattern responds more to maintain the local status quo, than to compete in the international market.
\end{abstract}

Keywords: parochial mindset, subnational policy, foreign trade, NAFTA, Mexico.

Artículo recibido el 29 de mayo de 2018 y dictaminado el 5 de noviembre de 2018.

1. Catedrático Conacyt-CIESAS. Consorcio de Investigación y Diálogo sobre Gobierno Local (Cidiglo). Prolongación Dr. Ángel Leaño \#1595, Colonia El Tigre, Zapopan, Jalisco, México. ORciD: http:// orcid.org/0000-0002-2473-5648. Correo electrónico: acanez@conacyt.mx 


\section{Introducción}

Los valores de los votantes, y no el descontento por la desigualdad económica, fue lo que determinó el voto en favor de la salida de Reino Unido de su lugar dentro de la Unión Europea, fenómeno llamado Brexit (Kaufmann, 2016). Personas con valores reaccionarios, en contra de la apertura y globalización son los que han impulsado los populismos nacionalistas en distintos países, donde estos valores coinciden con actitudes antiinmigrantes y proautoritarias, desconfianza en organismos internacionales, y conciencia de una ubicación ideológica extrema de izquierda o derecha (Inglehart \& Norris, 2016). Por ejemplo, el caso del actual Gobierno estadounidense de Donald Trump, quien pese a liderar una nación cuyo sistema económico está basado en el comercio internacional, ha "cerrado" la política de comercio exterior debido a ideas nacionalistas que toman como modelo una recreación del individuo estadounidense y del "antiguo esplendor" de Estados Unidos, excluyendo a grupos y sectores económicos que considera se han beneficiado de su país sin tomar en cuenta las ganancias económicas y sociales que le han proporcionado estos mismos grupos y sectores. Esta tendencia a encerrarse bajo discursos nacionalistas ha ido creciendo, tanto en países desarrollados como en emergentes, donde se culpa a la apertura como una de las causas de los problemas que enfrentan.

Un evento paradigmático reciente es la crisis financiera de 2009, cuando los mercados trascendieron las regulaciones nacionales y crearon vacíos de gobernanza, el resultado fue un rotundo fracaso del mercado e inestabilidad económica (Rodrik, 2013). Después de esta crisis, la relevancia y pertinencia de las políticas públicas para regular el mercado es prácticamente respaldada por todos los ángulos del espectro ideológico mundial, tanto de académicos como de políticos (Stiglitz, Yifu \& Monga, 2013). No obstante, en la realidad de las negociaciones de tratados internacionales de comercio, la política "politics" prevalece y los actores más fuertes imponen sus intereses, dejando a los jugadores más débiles fuera de los beneficios de dichos tratados. Por lo tanto, la hechura de las políticas comerciales, tanto internacionales como locales, debe ser revisada con más atención, para que no sólo sean producto de la imposición de los intereses de unos cuantos (Stiglitz, 2006).

Una de las causas por la que los países emergentes no ganan ventaja en las negociaciones internacionales es la corrupción, la cual ha sido un lastre y para combatirla los actores deben cambiar sus modelos mentales. Este cambio 
de mentalidad debe originarse a través de la democratización en la hechura de políticas públicas, con el fin de combatir las fallas de mercado y las fallas de gobierno. Esto por medio de la incorporación de diversos sectores en la hechura de políticas y evitar la tentación de que un grupo reducido las diseñe (Stiglitz, 2002).

La interdependencia es lo que predomina en el mundo globalizado contemporáneo; por lo tanto, los análisis académicos deben evitar simplificaciones como la idea de que los países sólo buscan intereses nacionales propios, porque son los gobiernos y las empresas las que empujan hacia sus intereses, algunas veces sin tomar en cuenta los intereses nacionales. Asimismo, las interacciones entre los países y grupos económicos no son relaciones de total dominación de unos hacia otros, sino que se trata también de vínculos interdependientes, donde incluso los actores más débiles pueden producir costos a los más poderosos (Keohane \& Nye, 2012). Entonces, se requiere abrir la caja negra de las estructuras y procesos de gobernanza, internacionales y nacionales, para comprender mejor las relaciones de interdependencia entre actores que buscan obtener sus propios intereses, mismos que influyen en la elaboración de las políticas de comercio internacional.

La investigación en torno a la hechura de políticas de comercio exterior debe dejar de lado simplificaciones y generalizaciones, para pasar a explorar las circunstancias específicas que inciden positiva o negativamente en el diseño y la eficacia de sus instrumentos. De hecho, en la literatura existen pocos estudios acerca de la injerencia de los actores subnacionales, públicos y privados, en el comercio y política exterior (Kukucha, 2008; Ventura-Dias, 2004). Otro problema ha sido que los analistas asumen comportamientos y no estudian cuáles ocurren en realidad (Buffie, 2001). En este contexto, con el presente artículo se pretende explorar el tipo de mentalidad que tienen actores subnacionales en la hechura de políticas de comercio exterior en el caso del estado de Sonora, México.

En este caso de Sonora existen diversos estudios previos acerca de la mentalidad de los empresarios en torno al comercio internacional, donde desde hace tiempo ha existido una fuerte clase empresarial ligada íntimamente a la clase política y a los subsidios gubernamentales (Guadarrama, 1987). Esta intimidad entre gobierno y empresarios sonorenses se ha explicado durante muchas décadas por una amalgama entre lo informal y lo privado, donde las redes de parentesco han ejercido un papel relevante en la imposición de los intereses privados sobre los intereses públicos (Almada, 2010). En este 
contexto, en el presente documento se pretende explorar el estado actual del vínculo entre la mentalidad de los actores y la hechura de la política de comercio exterior, para el caso específico de Sonora.

La estructura del presente trabajo se divide en cinco partes. En la primera, se presenta un breve panorama de las políticas de comercio exterior en entidades subnacionales. Después, se argumenta en torno a la mentalidad localista como obstáculo para la hechura de políticas de comercio exterior efectivas. En la tercera parte se presenta el caso de Sonora, México. Después, se describe una nota metodológica acerca de los criterios utilizados en la realización de las entrevistas semiestructuradas. En la quinta parte se presenta una reflexión en torno a la mentalidad localista en un entorno globalizado. Al final, se presentan las conclusiones.

\section{Las políticas de comercio exterior en entidades subnacionales}

El comercio exterior de las entidades subnacionales mexicanas está muy ligado al Tratado de Libre de Comercio de América del Norte (TLCAN); si ponemos este tratado en la balanza, tiene resultados positivos y negativos para México. Dentro de los positivos está el incremento de los flujos de comercio e inversión. Por ejemplo, las exportaciones de México a Estados Unidos y Canadá se multiplicaron por siete de 1993 a 2013, y las importaciones mexicanas se incrementaron en cuatro y ocho veces, respectivamente. Sin embargo, actualmente estos flujos se han desacelerado considerablemente durante los últimos 10 años, lo cual se torna en una situación desafiante. Por otra parte, dentro de los resultados negativos del TLCAN para México se encuentran el bajo crecimiento económico y menores oportunidades de empleo. De hecho, el TLCAN no parece ser la mejor estrategia para México, ya que los países de Sur y Centro América tuvieron mejor desempeño económico en los últimos 20 años. En resumen, el TLCAN ha traído ganancias en flujos de comercio e inversión, pero no se ha traducido en mejores empleos y salarios, reducción de la pobreza o reducción de la migración, como argumentaron inicialmente (Esquivel, 2014).

De acuerdo con Vázquez y Bocanegra (2015), el sector externo mexicano actual tiene un "brillo artificial" porque las cifras parecen más alentadoras que lo que realmente son. Es decir, más de la mitad de las exportaciones mexicanas son del sector maquilador y este sector importa la mayoría de los insumos de producción, utilizando sólo la mano de obra mexicana para después reexportar las mercancías con valor agregado. Un aspecto central del aparente éxito del 
sector externo mexicano es la baja competitividad de los empresarios locales para integrarse en las cadenas globales de producción. De hecho, el balance actual del TLCAN permite reforzar la idea de que un desarrollo exógeno requiere de fortaleza del mercado endógeno, ya que los beneficios no se producen de manera automática con la firma de tratados comerciales.

Por ello, las políticas públicas subnacionales de comercio exterior deben enfocarse en gestionar y formar parte de redes internacionales. Es decir, no sólo se deben tomar en cuenta los actores y recursos locales, sino también deben visualizar actores y recursos globales (Borja, Castells, Belil \& Benner, 1997).

Por su parte, buena parte de la discusión en torno a la inserción de las regiones en el mundo globalizado se enfocan en el concepto de competitividad, donde dicho concepto es amplio e incluye muchas variables, entre las que destacan las ventajas geográficas y económicas. Sin embargo, es necesario profundizar en el estudio de las características político-culturales de las regiones (Vázquez \& Hernández, 2001).

En este sentido, se requieren más estudios acerca del rol de los estados en la inserción económica de las regiones en el mundo globalizado. En específico, fortalecer la capacidad de innovación de los gobiernos estatales para contar con políticas públicas más efectivas (Tarr, 2008). La tendencia actual de innovación en los gobiernos locales apunta al paradigma de la nueva gobernanza, ya que las nuevas formas de gestionar lo público se dan en las interacciones entre los sectores público, privado y social. Es decir, innovar las prácticas y paradigmas de los actores involucrados, sin pensar que la innovación será el producto de una ley, programa o decreto (Klijn \& Koopenjan, 2006).

En los últimos años las políticas de comercio exterior han ido dejando atrás el enfoque tradicional, basado en la gestión centralizada en favor de la industria, el impulso a grandes proyectos y al financiamiento de las empresas; para dar entrada a un nuevo enfoque basado en la promoción de múltiples factores endógenos de cada región a través de una gestión descentralizada, así como el impulso en la formación de redes de cooperación de empresarios, gobiernos, universidades y demás organizaciones interesadas (Vázquez, 2005). Esto con la finalidad de mejorar los recursos y potenciales locales, así como las oportunidades derivadas del contexto global (Alburquerque, 2002). Son precisamente estos procesos de dirección de los esfuerzos de distintos actores hacia un objetivo común a lo que se le llama gobernanza (Aguilar, 2006), donde los esquemas interdisciplinarios son esenciales para enfrentar la creciente incertidumbre y complejidad actual (Rosenau, 1997). 
La relación entre las políticas públicas locales y las empresas de vocación internacional es una de las claves del comercio exterior para crear desarrollo local, donde se busca la generación de condiciones favorables para la productividad y competitividad, dejando atrás los subsidios y favores políticos que proveen los gobiernos a las empresas para atraer inversiones (Borja et al., 1997). Son estas políticas subnacionales las que pretenden generar condiciones favorables, principalmente en las pequeñas y medianas empresas, para fomentar las exportaciones e importaciones estratégicas para el desarrollo local (Calvento, 2016). Para ello, estas políticas subnacionales deben tener continuidad, ya que los beneficios de las estrategias se observan en el mediano y largo plazos, donde dichas estrategias deben estar respaldadas por recursos humanos altamente calificados y en la utilización de recursos científicos y tecnológicos, tanto en el gobierno como en las empresas (Dabat, 2002). En este sentido, en la medida que el gobierno, como organizador, y los empresarios, como actores interesados, perciban que sus intereses se encuentran atravesados por las dinámicas globales, las políticas públicas subnacionales tendrán una visión más internacional (Rodrik, 2013).

El presente artículo se basa en lo expuesto por Acemoglu y Robinson (2012), quienes afirman que las comunidades son pobres porque han sido gobernadas por élites que han dirigido políticas en beneficio propio a costa de la mayor parte de la población. En cambio, la pluralidad y la apertura son características esenciales en la formación de instituciones que han promovido desarrollo económico en comunidades prósperas, que se han caracterizado por tener mecanismos democráticos más efectivos. Entonces, este argumento apunta a que existe una cultura que moldea la elaboración de políticas públicas en cada comunidad. Pero, ¿mediante qué conceptos analizamos dicha cultura? En este estudio exploratorio propongo enfocarse en el estudio de la mentalidad de los actores, donde la hipótesis principal es que la mentalidad localista es un elemento cultural que afecta negativamente la hechura de políticas subnacionales de comercio exterior.

\section{La mentalidad localista como obstáculo para la hechura de políticas de comercio exterior efectivas}

La mentalidad global es una de las cualidades esenciales de los líderes empresariales contemporáneos, mientras que la mentalidad localista representa una debilidad en los negocios internacionales (Walsh, 2016). Los líderes con mayor 
éxito en las empresas internacionales son quienes, por sus características, están dispuestos a aprender constantemente, quienes tienen una mentalidad global, flexible, curiosa y con múltiples marcos de pensamiento para adaptar distintas maneras de pensar de otras personas (Cseh, Davis \& Khilji, 2013). De esta manera, la hechura de políticas de comercio exterior requiere de un sector empresarial activo y abierto, ya que una de las características más relevantes para que las empresas aprovechen las oportunidades globales es el respeto a la diversidad cultural (Herbert, 2001).

El ser cosmopolita — ciudadano del mundo con mentalidad global- es una empresa solitaria, sin las comodidades, calidez y seguridad que otorga el formar parte de una mentalidad localista. Este ciudadano no tiene un refugio, más que la convicción por la humanidad misma; en cambio el patriota cuenta con el respaldo de su discurso de estar en favor de los cercanos. Los retos contemporáneos requieren pensar las políticas públicas con una mentalidad global, porque al venerar la realidad local como un dios, se corre el riesgo de maldecirla (Nussbaum, 1999). Sin embargo, esta mentalidad global no significa vaciarse de tradiciones locales, sino respetar y pensar en las tradiciones de los otros, porque se corre el riesgo de la imposición de un solo sistema universal de valores, que niegue la diversidad de las culturas humanas (Butler, 1999). No obstante, cuando se pierde la ética y se tiende a los dos extremos, tanto cosmopolitismo como patriotismo, son problemáticos (Falk, 1999). En este sentido, en el presente artículo se argumenta acerca de las ventajas que tiene adoptar una mentalidad global en las políticas de comercio exterior, sin pretender que esto sea una panacea.

El concepto de mentalidad supone un ser humano de racionalidad limitada, quien tiene limitaciones para procesar la ambigüedad y complejidad de la cantidad de información que nos rodea (Simon, 1962). Entonces, el término mentalidad hace referencia a los filtros cognitivos con los que se interpreta la información que recibimos y que están compuestos por las experiencias pasadas. Mientras la nueva información confirma nuestra manera de pensar, la mentalidad se refuerza; y cuando dicha información es inconsistente con nuestra mentalidad, pueden suceder dos cosas: se rechaza o se cambia la mentalidad. El cambio se produce cuando se tiene conciencia del tipo de mentalidad que posee cada quien, es decir, cuando somos autoconscientes y autocríticos. En ese sentido, la formación de una mentalidad colectiva es producto de la interacción de varias mentalidades individuales, y lo que determinará el mantenimiento de una mentalidad colectiva en particular es la 
distribución de poder e influencia entre los miembros del colectivo (Gupta \& Govindarajan, 2002).

La mentalidad localista tiende a ser más intensa en la clase empresarial que en la clase trabajadora, ya que los dueños del capital local tienen motivos para considerar al extranjero como competidor suyo. Aunque, también la clase trabajadora tiende a ensalzar el nacionalismo y culpar a los extranjeros de los males locales. En cambio, la mentalidad internacional permite identificar problemas y oportunidades sin prejuicios de intereses localistas y habilita el surgimiento de nuevos ganadores, recomponiendo la estructura empresarial local. A pesar de que Betancourt (1934) escribió esto hace 84 años, considero que su pensamiento sigue vigente, debido a las representaciones de ultranacionalismo que promueven actualmente diversos jefes de gobierno.

La mentalidad localista puede representar una especie de miopía porque sólo se ve una parte del mercado internacional, dejando fuera realidades económicas que no se reflejan en el espacio local (Eagleton, 2011). Sin embargo, la mentalidad localista representa una aprobación interna de las personas de la comunidad en cuestión, ya que así se demuestra lealtad a las tradiciones locales que otorgan identidad. En cambio, cuando un individuo muestra lealtad hacia Estados o entes supranacionales, se le considera como traidor (Soto, 2015). En este sentido, el predominio de patrones localistas significa agradar a la comunidad local y representa mantenerse en una zona de bajo riesgo e incertidumbre, que pudiera ser modificada por el contacto internacional (García del Junco, Álvarez \& Reyna, 2007). Es importante aclarar que ni toda mentalidad localista es negativa, ni toda mentalidad internacional es positiva. Existen matices. Lo que se argumenta en este artículo es la serie de circunstancias que hacen que la mentalidad localista sea un obstáculo para el comercio exterior en una entidad subnacional. De hecho, algunas personas tienden a pensar que la globalización restará soberanía a los Estados nacionales (Soto, 2015). Pero acaso ¿no se resta soberanía también cuando un pequeño grupo de nacionales mantiene un statu quo basado en la desigualdad y en la acumulación de capital? ¿Existe alguna diferencia sustantiva en el hecho de que la dominación económica la ejerza un grupo de nacionales o un grupo de extranjeros? Yo considero que no existe diferencia sustancial, ya que la soberanía democrática debe ser para las mayorías, para el pueblo, no para un grupo reducido de personas, independientemente de la nacionalidad que tengan.

En esta relación entre lo global y lo local es importante tener una perspectiva global, pero también defender los valores locales que identifican a 
cada comunidad. En este sentido, el abrirse al mundo no significa ceder ante la hegemonía de valores universales. No obstante, el otro extremo de lugares desconectados de lo global representa un riesgo de desintegración social porque los centros urbanos globales tenderán a relegar a estos grupos locales cerrados. Para contrarrestar esto es necesario que las políticas públicas incorporen a las distintas regiones y no tiendan a centralizar las acciones sólo en las grandes metrópolis. Esto aplica tanto para los gobiernos nacionales como para los gobiernos provinciales o subnacionales (Borja et al., 1997).

Un ejemplo multicitado es el de ambos Nogales, dos ciudades divididas por un muro en la frontera entre México y Estados Unidos; estas ciudades comparten la misma geografía, clima, una parte importante de la población son incluso de la misma raza. Entonces, ¿por qué una ciudad tiene mayor prosperidad que la otra? Si incluso ciudadanos mexicanos de Nogales, Sonora, cruzan a trabajar a la ciudad estadounidense de Nogales, Arizona.

Como afirman Acemoglu y Robinson (2012), son las instituciones económicas las que determinan la diferencia entre prosperidad y pobreza, al mismo tiempo que éstas son producidas por las instituciones políticas. Yo añado el siguiente argumento: la mentalidad de los actores involucrados moldea también la elaboración de políticas públicas. Para explorar esta hipótesis, se elige el caso de la política de comercio exterior en la entidad subnacional de Sonora, México.

El criterio de selección de Sonora es porque es un estado fronterizo con Estados Unidos y con alta intensidad de comercio exterior, ya que se encuentra entre los cinco estados con mayores exportaciones como porcentaje del PIB estatal (Instituto Mexicano para la Competitividad [IMCO], 2016). El ser un estado fronterizo con Estados Unidos beneficia a la hipótesis exploratoria contraria a la mentalidad localista, debido a los múltiples intercambios comerciales y culturales que existen en la región fronteriza, lo cual supone la existencia de una mentalidad global o abierta.

\section{El caso de Sonora, México}

La historia del comercio exterior en Sonora se circunscribe en cuatro episodios. El primero se refiere a la época de los mercaderes europeos, del año 1800 a 1850, quienes ingresaban sus productos por el Puerto de Guaymas, los dejaban a crédito para cobrar después de la venta al menudeo. El segundo episodio se refiere a la época de los chinos, desde el año 1850 hasta 1930, quienes 
llegaron a Sonora para trabajar en la construcción del ferrocarril, quienes pronto monopolizaron el comercio local gracias a su capacidad de trabajo y ahorro. Sin embargo, los comerciantes locales presionaron al gobierno estatal de Rodolfo Elías Calles para la expulsión de los chinos, quien finalmente lo hizo (Bocanegra, 2007).

El tercer episodio es la expansión agrícola y comercial, del año 1930 a 1975, cuando se formó una nueva clase empresarial sonorense. Estos empresarios, quienes comerciaban en las grandes ciudades, importaban productos del resto de la república y desplazaron a los productos locales. Por último, el cuarto episodio se refiere a las empresas transnacionales, de 1980 hasta nuestros días, quienes quitaron el monopolio del comercio a los empresarios locales. En este periodo se acentuó la inercia de desarrollar la costa, más que la sierra, lo que ha impulsado que la población y la economía se concentren en las ciudades costeras (Bocanegra, 2007).

El punto fuerte del comercio exterior actual en Sonora está basado en la armadora de automóviles Ford y en la minería. Ambos tienen la característica de ser intensivos en mano de obra y estar ligados a empresas transnacionales que importan la mayoría de sus insumos para después exportarlas con valor agregado para el mercado internacional (Moreno, 2012). La vulnerabilidad del sector externo sonorense es un asunto a destacar a lo largo de su historia, debido a la ausencia de estrategias de articulación de su mercado interno con el externo.

Actualmente el marco jurídico-administrativo del comercio exterior en Sonora obedece a dos enfoques: a) el de finanzas públicas, y b) el de desarrollo económico. El enfoque de finanzas públicas gira en torno a la Coordinación Ejecutiva de Verificación al Comercio Exterior (CEVCE), donde ésta es producto de la coordinación entre el Gobierno federal de México y el Gobierno subnacional de Sonora. En el año 1996 se firmó este convenio en el que dota a Sonora de la facultad de verificación fiscal vehicular y auditoría fiscal para ciertos impuestos. En el año 2004 se firmó el Anexo 8, que actualiza facultades que siguen enfocadas en combatir la evasión fiscal por contrabando de mercancías (Coordinación Ejecutiva de Verificación al Comercio Exterior [CEVCE], 2013).

Por su parte, el enfoque de desarrollo económico gira en torno al Consejo para la Promoción Económica de Sonora (COPRESON). Este Consejo tiene como propósito fomentar las exportaciones y vincular a los actores públicos y privados para impulsar una política de desarrollo de las regiones de Sonora y atracción de inversión extranjera directa. La organización directiva de la COPRESON se divide en un Consejo Estatal y seis Consejos regionales. El Con- 
sejo Estatal es presidido por el gobernador; los secretarios de Economía y de Turismo participan como vocales gubernamentales. Además, se incorporan los representantes de las principales cámaras empresariales como vocales del sector privado. Asimismo, se integran los presidentes de los seis Consejos regionales y también un presidente municipal como representante de los presidentes municipales de cada una de las seis regiones. También se integran dos representantes del sector sindical y los demás invitados del gobernador aprobados por el Consejo Estatal. Paralelamente, existe también el Consejo de Desarrollo Económico, el cual se integra de manera similar que el Consejo Estatal (Ley de Fomento Económico del Estado de Sonora, 156 H. R., 2017).

El común denominador en las últimas tres administraciones estatales es que no se pueden evaluar con precisión sus resultados. A pesar de que los informes de gobierno presentan datos estadísticos, estos se limitan a cuantificar acciones de gobierno, más que a evaluar la efectividad de los resultados obtenidos. Por ejemplo, los informes resaltan el número de reuniones, visitas y viajes, pero no se explica en qué medida estas acciones ayudaron a mitigar las causas de los problemas públicos. Considero que este paradigma de rendición de cuentas está relacionado con el énfasis en justificar la cantidad de horas trabajadas y recursos utilizados, dejando fuera el enfoque de informar, explicar y justificar la calidad de dichas acciones, es decir, los informes de gobierno han respondido más a un modelo de cumplir con procedimientos, que a un sistema de alcanzar resultados.

La apuesta actual del Gobierno de Sonora es continuar apoyando los sectores agroindustria, manufactura y aeroespacial mediante incentivos fiscales y no fiscales para las grandes empresas. Por ejemplo, en 2016 se apoyaron cuatro empresas que generan alrededor de 1,500 empleos en los municipios de Guaymas, Hermosillo y Nogales. Además, el Gobierno estatal aprovechó un programa federal y apoyó el establecimiento del Centro Regional de Investigación, Desarrollo Tecnológico e Innovación para el Sector Aeroespacial, el cual estará situado en el Instituto Tecnológico de Hermosillo. Por su parte, existe otra apuesta fuerte en la atracción de inversión extranjera para la generación de energías limpias, aprovechando la intensa luz solar que hay en todo el año en Sonora (Informe Economía Sonora, 2016). Cabe destacar que a pesar de lo escrito en los planes e informes, existe un constante rompimiento de la política de comercio exterior cada sexenio, ya que hay rotación de personal y cambios de prioridades en las oficinas gubernamentales. 
Particularmente, si se compara el ambiente empresarial en Sonora respecto al resto de los estados mexicanos, ocupa la posición 12 de 32 en el último reporte de Doing Business; Sonora presenta sus mejores números en la rapidez para obtener un permiso de construcción; sin embargo, sus peores números están en el bajo cumplimiento de contratos, es decir, el tiempo y costo que representa promover un juicio mercantil (Banco Mundial, 2016).

De esta manera podemos visualizar un bosquejo del caso sonorense, un breve recuento histórico de las tensiones y conflictos en torno al mercado local y su posición en dos contextos más amplios, el nacional y el internacional.

\section{Nota metodológica}

La estrategia metodológica del presente documento se basa en análisis documental y entrevistas semiestructuradas realizadas a expertos. Dentro del análisis documental, se revisó la normatividad en materia de comercio exterior en Sonora. También se analizaron los planes estatales de desarrollo, desde 1997 a la actualidad, con el fin de conocer la evolución del planteamiento del rol del Gobierno estatal como promotor del comercio exterior. Se toma el año de 1997 como punto de partida porque fue el primer Gobierno estatal que inició un sexenio con el TLCAN en funcionamiento. Por otra parte, se realizaron entrevistas semiestructuradas a nueve expertos con la finalidad de explorar las prácticas de los actores públicos, privados y sociales.

El criterio de selección del contenido de las respuestas de los entrevistados fue la coincidencia, es decir, la interpretación de las entrevistas se realizó una vez terminadas las nueve, y se buscó en qué temas coincidieron el mayor número de entrevistados. Considero que mostrar las coincidencias en las respuestas es un criterio adecuado para un estudio exploratorio. Una limitación del presente artículo es que no se profundizó en los matices cuando los entrevistados tenían posiciones contrarias; sin embargo, eso es parte de un estudio posterior; además, los puntos en que divergieron no contradicen a los que coincidieron. Fue en los detalles y en las estrategias de solución en lo que más divergieron las posiciones de los entrevistados. Por ejemplo, en general los entrevistados del sector universitario coincidieron en que las estrategias gubernamentales y los subsidios están mal dirigidos. Por su parte, los entrevistados del sector gubernamental coincidieron en que los empresarios no están dispuestos a invertir en ciencia y tecnología para ser más competitivos. Mientras que los entrevistados del sector empresarial coincidieron en que 
el Gobierno debería tener un rol más activo en la estrategia subnacional de comercio exterior, así como poner más atención en la regulación de sindicatos, ya que el abuso de líderes sindicales obstaculiza la atracción y retención de inversiones. Pese a estas diferencias en determinar la mejor estrategia de solución, todos los entrevistados coincidieron en que el problema se encuentra en una cultura empresarial y gubernamental cerrada y tradicional.

La estrategia de selección de los expertos fue por "bola de nieve". El punto de partida de las entrevistas para el sector público fue la Secretaría de Economía del Gobierno del estado de Sonora; para el sector privado fue la sección Sonora de la Confederación Patronal de la República Mexicana; y para el sector universitario el punto de partida fue el Departamento de Economía de la Universidad de Sonora. Todos ubicados en la ciudad de Hermosillo, capital de Sonora y sede de los poderes estatales. A partir de ahí, se desplegaron el resto de las entrevistas a expertos, tomando en cuenta la saturación de respuestas como el criterio para finalizar con este método. Durante los meses de mayo y junio del año 2017 se entrevistó a nueve actores relacionados directamente con el comercio exterior en Sonora, tres del sector público, tres del sector privado y tres del sector universitario. Del sector público se entrevistó a un funcionario de la delegación federal de la Secretaría de Economía, a un funcionario de la Comisión para la Promoción Económica del Estado de Sonora, y a un funcionario de la Coordinación Ejecutiva de Verificación al Comercio Exterior. Del sector privado se entrevistó a un integrante de la mesa directiva de la Confederación Patronal de la República Mexicana, sección Sonora; a un integrante del Consejo Nacional de la Industria Maquiladora y Manufacturera de Exportación, sección Sonora; y a un integrante de la Cámara Nacional de la Industria de Transformación, sección Sonora. Del sector universitario, se entrevistaron tres profesores adscritos al posgrado de Comercio Exterior de la Universidad de Sonora. A continuación, en la tabla 1 se presenta el cuestionario de entrevistas, que es el mismo para todos los entrevistados. 
Tabla 1

Cuestionario de entrevista dirigido a expertos en materia de comercio exterior en el estado de Sonora

Modelo de preguntas aplicadas

¿Cómo ve la situación del comercio exterior en Sonora?

¿Qué está funcionando?

¿Qué no está funcionando?

¿Cuál es el rol del Gobierno estatal en materia de comercio exterior?

¿Cuál es el rol del sector privado en materia de política de comercio exterior?

¿Cuál es el rol de las universidades en la política de comercio exterior?

¿Cómo se pudiera mejorar la política de comercio exterior en Sonora?

¿Qué obstaculiza la realización de estas mejoras?

Lo que se buscó obtener con las entrevistas fue conocer la posición de cada uno de los expertos en materia de comercio exterior. A partir de ahí, se compararon las respuestas e interpretaron los resultados para conocer, de manera exploratoria, el tipo de mentalidad que consideran los entrevistados predomina en los principales actores que participan en la hechura de la política subnacional de comercio exterior.

\section{Mentalidad localista en un entorno globalizado}

Los expertos que fueron entrevistados coincidieron en que es precisamente el tipo de mentalidad el principal factor que explica la hechura de la política de comercio exterior en Sonora. En el sector público predomina un aparato burocrático dirigido, predominantemente, por patrones de decisión de aparatos políticos (Cáñez, 2011). Es decir, el patrón de decisión en torno al reclutamiento, selección y promoción del personal encargado de dirigir la política de comercio exterior se explica por la lealtad y cercanía al grupo político en turno. Esto significa que a pesar de las capacidades técnicas con las que cuentan los burócratas, los motivos políticos predominan.

La mayoría de los entrevistados mencionaron que la falta de un sistema profesional de carrera es un gran pendiente. Por ejemplo, mencionan que es muy evidente la diferencia en el perfil del promotor del comercio exterior sonorense con el estadounidense. Siete de los nueve entrevistados mencio- 
naron la falta de estrategia gubernamental como uno de los aspectos que más afectan al comercio exterior en Sonora. Un entrevistado, que tiene más de 20 años de experiencia en misiones comerciales, comentó que cuando un empresario sonorense solicita ayuda en alguna oficina estadounidense de Arizona, éste recibe toda la información técnica necesaria para invertir. En cambio, cuando un estadounidense acude a una dependencia gubernamental sonorense, éste recibe información parcial, lo cual no promueve la certidumbre para invertir. Curiosamente, el burócrata sonorense se preocupa más por la atención personal que por la atención profesional. Esto es, se preocupa por atender al inversionista, desde ir al aeropuerto y llevarlo a comer. También, otorgar regalos y souvenirs como bacanora, machaca y carne seca, etc. Incluso, en ocasiones se les invita a paseos en yate en la marina de San Carlos.

Este ejemplo es útil para explorar la naturaleza del ambiente empresarial, burocrático y político en Sonora. A pesar de que la intensa competencia económica internacional es un hecho evidente, las prácticas tradicionales siguen presentes en gran parte de los actores sonorenses. Por ejemplo, los nueve entrevistados coinciden en que la cultura de inversión de muchos empresarios sonorenses es cerrada. Es decir, los empresarios prefieren hacer negocios con sus amigos y parientes que hacerlo con empresas fuera de Sonora. En lugar de buscar al mejor socio de México o del mundo para un negocio, buscan al pariente o amigo, aunque éste no sea experto en la materia. Cabe señalar que los entrevistados se refieren a la mayoría de empresarios; también mencionaron que existen excepciones y tres entrevistados destacaron el caso de la empresa Caffenio, que hizo una alianza comercial con Oxxo del grupo FEMSA de Monterrey. Además, ocho de los nueve entrevistados coincidieron en que el ambiente burocrático y político también es cerrado, ya que existe una profunda desvalorización del conocimiento científico en la hechura de políticas públicas.

Estas entrevistas exploratorias apuntan a que existe una mentalidad cerrada en la elaboración de la política de comercio exterior en Sonora, cerrada en cuanto a una visión basada en tradiciones regionalistas, así como también cerrada en cuanto a la falta de promoción y generación del conocimiento científico local, ya que se reproducen ideas del exterior y existe un déficit de innovación, tanto en el sector público como en el privado. Este ambiente cerrado puede ser explicado, en parte, por la ausencia de la meritocracia en muchas de las actividades empresariales, políticas y burocráticas. 
Donde la meritocracia no predomina, la competencia no puede florecer. En este sentido, el comercio exterior es un sector que requiere apertura y competencia para ser efectivo. Entonces, la política de comercio exterior subnacional debería estar enfocada en promover la apertura y la competencia. Sonora tiene un freno importante a la competitividad debido a la preferencia de las instancias gubernamentales por contratar proveedores locales. No se realizan análisis de mercado sobre la materia a licitar y se tiene amplia discrecionalidad para modificar los requisitos de las licitaciones (Comisión Federal de Competencia Económica [COFECE], 2016). Asimismo, Sonora destaca como uno de los estados con mayor incidencia de corrupción, superado sólo por Estado de México, Sinaloa y Chihuahua (Instituto Nacional de Estadística, Geografía e Informática [INEGI], 2015).

La calidad de las decisiones en materia de comercio exterior en Sonora parece estar marcada más por la tradición y mentalidad localista, que por la evidencia científica y la apertura a la competencia internacional. Por ejemplo, siete entrevistados coinciden en que el empresario promedio sonorense está acostumbrado a recibir subsidios para consultoría; por lo tanto, no valora el conocimiento técnico externo a su empresa, ya que el servicio se les hace muy caro. Además, estos siete entrevistados coinciden en que el empresario promedio tiene fuertes tradiciones como mantener el mismo broker de la familia, aun cuando se tengan mejores ofertas en el mercado. Los empresarios llegan a conocer tanto al broker, que lo consideran como su amigo íntimo.

Por su parte, la burocracia encargada de promover el comercio exterior en Sonora tiene alta rotación de personal debido a los cambios de partido en el Gobierno. Es decir, cada seis años hay una rotación de personal de los puestos directivos y se pierde la confianza que se había cultivado con burocracias y empresarios extranjeros. Además, cinco entrevistados coinciden en que estos burócratas temporales no desarrollan un perfil de resolución de problemas para el comercio exterior e inversión extranjera, sino que desarrollan más un perfil de estilo diplomático. Entonces, no existe un aprendizaje institucional que desarrolle una memoria y mejores prácticas. Esto a pesar de que el burócrata promedio tiene las credenciales necesarias para desarrollar su comisión; sin embargo, carece de un sistema profesional que valore el aprendizaje institucional.

Otro problema relacionado con el predominio de una mentalidad localista es la falta de un sistema de evaluación de la política de comercio exterior, donde se promueva el monitoreo de la atracción y retención de inversión 
extranjera. Es normal que el Gobierno estatal informe de los montos de inversión extranjera atraídos; sin embargo, omite el reconocimiento de los montos que se han ido.

En el presente estudio se considera que el problema de política pública de fondo en materia de comercio exterior en Sonora es que el paradigma de comercio tradicional cerrado no es compatible con los requerimientos contemporáneos de apertura a la competencia internacional. Las causas de este problema son los patrones de decisión de empresarios y burócratas, basados en una mentalidad localista. Estos toman decisiones más basados en la lealtad a los grupos de confianza, que movidos por crecer en la industria (empresarios) o por resolver problemas públicos (burócratas). En este sentido, el problema de estar cerrado mentalmente a una economía global tiene como consecuencia que Sonora sea visto como un lugar maquilador estratégico. Entonces, las inversiones y salarios no se maximizan para beneficio de los actores sonorenses, sino que se van a otros estados y países.

De esta forma, el común denominador de estas situaciones problemáticas, que afectan la competencia e innovación, es la mentalidad y prácticas tradicionales localistas en un entorno que exige prácticas abiertas e internacionales. Es decir, las causas del problema parecen estar ligadas a la desincronización entre la mentalidad y prácticas localistas de empresarios y burócratas en relación con la mentalidad y prácticas abiertas que se requieren en una economía globalizada. En resumen, se trata de una falta de congruencia entre la necesidad de apertura y las rutinas cerradas.

En la actualidad el comercio exterior requiere apertura, no sólo en las fronteras geográficas sino también en las fronteras mentales. Como ejemplo de constante innovación en los esquemas mentales, las regiones innovadoras estadounidenses, especialmente en Silicon Valley, la mayoría del personal ocupado tiene grado de licenciatura o posgrado (Melville, Kaiser \& Brown, 2017). Esto indica que las industrias con enfoque científico y tecnológico aprovechan el talento humano como activo y no sólo como factor de producción, es decir, el recurso humano pasa de ser visto como maquilador a creador. Además, las industrias que utilizan mano de obra calificada ofrecen mejores salarios y prestaciones para los trabajadores.

Por último, ocho de los nueve entrevistados también coincidieron en que el grado de la mentalidad localista es mayor en Sonora en comparación con otras entidades subnacionales mexicanas. Por ejemplo, a diferencia de las zonas más industrializadas de México como el Bajío, Monterrey y Guadalajara, 
en Sonora los empresarios están más imbricados en la política, lo que impide que los empresarios sean un contrapeso real al Gobierno. Por ejemplo, dice un entrevistado que en Querétaro el sector empresarial exigió al Gobierno la permanencia de burócratas clave en la implementación de nuevas inversiones de la industria aeronáutica. También, el empresariado de Monterrey genera mucha presión sobre el Gobierno, que en ocasiones incluso el sector privado le pide al Gobierno que no intervenga y distorsione el ambiente de inversiones. Entonces, de manera exploratoria la mentalidad localista sin contrapesos parece ser el núcleo del problema en la hechura de la política de comercio exterior en Sonora.

\section{Conclusión}

El presente artículo aporta evidencia de un caso subnacional calificado de "intermedio", donde Calvento, Lorenzo y Herrero (2014) llaman a estudiar más casos que no estén ligados a megaciudades, con el fin de explorar las políticas subnacionales de comercio exterior fuera de grandes metrópolis. Asimismo, aporta evidencia al concepto de metagobernanza, el cual Kooiman (2005) entiende como los aspectos culturales y mentalidad de los actores que moldean las políticas públicas, donde dicho concepto es uno de los más desafiantes en su comprensión dentro de la teoría de la gobernanza y políticas públicas (Sorensen \& Torfing, 2009). En concreto, se propone el estudio del concepto de mentalidad localista como una manera de entender los obstáculos en la hechura de las políticas subnacionales de comercio exterior.

La lección que ofrece el caso de Sonora es que la intensa imbricación entre la clase política y la empresarial deprime la efectividad de la hechura de la política de comercio exterior, ya que no existen los contrapesos necesarios para tomar decisiones en competencia. El principal hallazgo es que la mentalidad localista de la mayoría de burócratas, empresarios y trabajadores sindicalizados predispone que las decisiones de política pública respondan más a mantener la competencia y el statu quo local, que a competir en el mercado internacional. Considero que los esfuerzos para mejorar las políticas de comercio exterior en Sonora requieren enfocarse más en cambiar la mentalidad que en seguir manejando sólo discursos de apertura.

Otro problema es que las soluciones de política pública no están amarradas a un esquema profesional, en donde la ciencia y tecnología sea el centro de gravedad. En general, los burócratas llegan a sus puestos por motivos políticos 
y existe una alta rotación de personal; los empresarios locales no invierten lo suficiente en el uso del conocimiento científico para desarrollar sus negocios. Por su parte, los centros de educación superior carecen de una vinculación efectiva entre sus egresados y la industria. Cabe señalar que existen excepciones a estos patrones predominantes.

Con base en este estudio exploratorio, propongo poner atención en que no es la falta de financiamiento el principal obstáculo para el comercio exterior en Sonora, sino la mentalidad que se resiste a valorar el mérito, la innovación y la competencia internacional. En suma, parece que los actores interesados están en una zona de confort, la cual no les exige asumir mayores riesgos y sacrificios con el fin de generar mayores ganancias. Los proyectos a largo plazo están ausentes y un grupo reducido de empresarios y políticos mantienen control de los incentivos para promover el surgimiento de nuevos empresarios locales. En Sonora predomina una mentalidad basada en la lealtad más que en el mérito, en la zona de confort más que en la innovación. Los efectos de este problema consisten en que se tiene una economía principalmente maquiladora que genera empleos con baja remuneración y productos con bajo valor agregado.

En resumen, considero que se debe transitar de la idea de "resolver problemas con dinero e infraestructura", hacia "resolver los problemas con inteligencia". En México hay numerosos ejemplos de cómo un mayor presupuesto no se traduce en mayor efectividad en las políticas públicas. Una agenda futura de investigación, en materia de la hechura de políticas subnacionales de comercio exterior, es comprender cómo sociedades con una mentalidad abierta y globalista generan mercados competitivos que producen un desarrollo más incluyente para los miembros de dichas sociedades. Así como encontrar los instrumentos de política pública que puedan revertir la tendencia de mentalidad localista.

\section{Referencias bibliográficas}

Acemoglu, D., \& Robinson, J. (2012). Por qué fracasan los países. Los orígenes del poder, la prosperidad y la pobreza. Barcelona: Deusto S. A. Ediciones. Aguilar, L. (2006). Gobernanza y Gestión Pública. México: Fondo de Cultura Económica.

Alburquerque, F. (2002). Marco conceptual y estrategia para el desarrollo local. Recife: BNDES/PNUD. 
Almada, I. (2010). De regidores porfiristas a presidentes de la República en el período revolucionario. Explorando el ascenso y la caída del 'sonorismo'. Historia Mexicana, 60(2), 729-789.

Banco Mundial (2016). Doing Business en México. Midiendo la calidady eficiencia de la regulación. Recuperado de http://www.doingbusiness.org/content/ dam/doingBusiness/media/Subnational-Reports/DB16-Sub-Mexico.pdf

Betancourt, R. (1934). Contra el localismo y el patrioterismo: por una mentalidad internacionalista. Recuperado de http://saber.ucab.edu.ve/bitstream/ handle/123456789/8097/rb047119341125.pdf?sequence=3

Bocanegra, C. (2007). El comercio en Sonora: una visión desde la perspectiva de su evolución. Estudios Fronterizos, 8(15), 73-97.

Borja, J., Castells, M., Belil, M., \& Benner, C. (1997). Local and Global. The Management of Cities in the Information Age. Londres: Routledge.

Buffie, E. F. (2001). Trade policy in developing countries. United States of America:

Cambridge University Press.

Butler (1999). La universalidad de la cultura. En Martha Nussbaum y Joshua Cohen (Eds.), Los límites del patriotismo. Identidad, pertenencia y ciudadanía mundial (pp. 59-66). Barcelona: Paidós

Calvento, M. (2016). La Política Internacional Subnacional: una propuesta para el abordaje del accionar contemporáneo en Argentina. Desafíos, 28(1), 297-334.

Calvento, M., Lorenzo, N., \& Herrero, M. (2014). Análisis teórico-empírico de la gestión internacional de los actores subnacionales: una propuesta de abordaje para las ciudades intermedias. CONfines de relaciones internacionales y ciencia política, 10(20), 35-58.

Cáñez-Cota, A. (2011). Patrones de decisiones en la acción gubernamental municipal: El caso de Hermosillo, Sonora. (Tesis de Maestría). Recuperada de Repositorio Digital CIDE. (Acceso abierto)

Comisión Federal de Competencia Económica-COFECE. (2016). Miscelánea de obstáculos regulatorios a la competencia. Recuperado de https://www. cofece.mx/cofece/images/Promocion/Miscelanea_Estatal_210916.pdf

Coordinación Ejecutiva de Verificación al Comercio Exterior (CEVCE). (2013). Manual de Organización. Secretaría de Hacienda de Sonora, Hermosillo.

Cseh, M., Davis, E., \& Khilji, S. (2013). Developing a global mindset: learning of global leaders. European Journal of Training and Development, 37(5), 489-499. doi:10.1108/03090591311327303 
Dabat, G. (2002). Políticas municipales de competitividad y comercio exterior. En G. Dabat (Coord). Desde Adentro. Políticas municipales de competitividad y comercio exterior. (pp.23-54) Buenos Aires, Universidad Nacional de Quilmes.

Eagleton, T. (2011). Por qué Marx tenía razón ¿Y si todo lo que siempre se le ha recriminado fuera falso? Barcelona: Ediciones Península.

Esquivel, G. (2014). El TLCAN: 20 años de claroscuros. Foreign Affairs Latinoamérica, 14(2), 7-16

Falk, R. (1999). Una revisión del cosmopolitismo. Martha Nussbaum (Coord). Los límites del patriotismo. Identidad, pertenencia y ciudadanía mundial. (pp. 1-11) Barcelona: Paidós.

García del Junco, J., Álvarez, P., \& Reyna, R. (2007). Características del Emprendedor de Éxito en la Creación de PYMES Españolas. Estudios de Economía Aplicada, 25(3), 951- 974.

Guadarrama, G. (1987). Empresarios y política: Sonora y Nuevo León, 1985. Estudios Sociológicos, 5(13), 139-168.

Gupta, A. \& Govindarajan, V. (2002). Cultivating a global mindset. The Academy of Management Executive, 16(1), 116-126. doi:10.5465/ ame.2002.6640211

Herbert, P. (2001). Creating a global mindset. Thunderbird International Business Review, 42(2), 187-200. doi: 10.1002/1520-6874(200003/04)42

Herbert, S. (1962). El comportamiento administrativo: estudio de los procesos decisorios en la organización administrativa. Madrid: Aguilar.

Informe Economía Sonora (2016). Informe de trabajo de la Secretaría de Economía en Sonora. Recuperado de http://www.economiasonora.gob.mx/ portal/descargas/se_informe_anual_2016.pdf

Inglehart, R. \& Norris, P. (2016). Trump, Brexit, and the rise of Populism: Economic have-nots and cultural backlash. Recuperado de https://faculty.uml. edu/sgallagher/Trump_Populism_Norris.pdf

Instituto Mexicano para la Competitividad (IMCO). (2016). Índice de competitividad estatal. Un puente entre dos Méxicos. Recuperado de https:// imco.org.mx/wp-content/uploads/2016/11/2016-ICE-Libro.pdf

Instituto Nacional de Estadística y Geografía (INEGI). (2015). Encuesta Nacional de Calidad e Impacto Gubernamental 2015. Recuperado de http:// www.beta.inegi.org.mx/programas/encig/2015/

Kaufmann, E. (07 de julio de 2016). It's NOT the economy, stupid: Brexit as a story of personal values [Mensaje en un blog]. Recuperado de http:// 
eprints.lse.ac.uk/71585/1/blogs.lse.ac.uk-Its\%20NOT\%20the\%20economy\%20stupid\%20Brexit\%20as\%20a\%20story\%20of\%20personal\%20 values.pdf

Keohane, R. O., \& Nye, J. S. (2012). Power and Interdependence (Cuarta edición). New York: Longman Pearson.

Klijn, E. \& Koopenjan, J. (2006). Public Management and Policy Networks: Foundations of a network approach to governance. Public Management: An International Journal of Research and Theory, 2(2), 135-158. doi:10.1080/14719030000000007

Kooiman, J. (2005). Gobernar en Gobernanza. En Agustí Cerrillo (Coord.), La Gobernanza Hoy: 10 textos de referencia. (pp.9-245). Madrid: Instituto Nacional de Administración Pública.

Kukucha, C. J. (2008). The provinces and Canadian foreign trade policy. Vancouver: University of British Columbia Press.

Ley de Fomento Económico del Estado de Sonora, 156 H. R. (2017).

Melville J., Kaiser, J. \& Brown, E. (2017). Silicon Valley Competitiveness and Innovation Project-2017 Report. A Dashboard and Policy Scorecard for a Shared Agenda of Prosperity and Opportunity. Recuperado de https://svcip.com/ files/SVCIP_2017.pdf

Moreno, A. (2012). Comercio exterior e inversión extranjera en México y Sonora a la hora de la apertura comercial. Un balance preliminar. Hermosillo: Pearson Educación.

Nussbaum, M. (1999). Patriotismo y Cosmopolitismo. En Nussbaum, M., \& Cohen, J. (Eds.), Los límites del patriotismo. Identidad, pertenencia y ciudadanía mundial (pp. 13-29). Barcelona: Paidós.

Rodrik, D. (2013). Gobiernos nacionales, ciudadanos globales. El Economista. Recuperado de http://www.eleconomista.es/firmas/noticias/4699214/03/13/Gobiernos-nacionales-ciudadanos-globales.html

Rosenau, J. (1997). Demasiadas cosas a la vez: La teoría de la complejidad y los asuntos mundiales. Nueva Sociedad, (148), 70-83.

Sørensen, E., \& Torfing, T. (2009). Making Governance Networks Effective and Democratic Through Metagovernance. Public Administration, 87(2), 234-258. doi: 10.1111/j.1467-9299.2009.01753.x

Soto, W. (2015). Subjetividades políticas y emergencia de ciudadanos globales. En Piedrahita, C .L., Díaz, A., \& Vommaro, P. (Comp.), Pensamientos críticos contemporáneos: análisis desde Latinoamérica (pp.177-187). Bogotá: CLACSO. 
Stiglitz, J. (2002). El malestar en la globalización. Madrid: Taurus.

Stiglitz, J. (2006). Making globalization work. New York: W. W. Norton and Company.

Stiglitz, J., Yifu, J., \& Monga, C. (2013). Introduction: The Rejuvenation of Industrial Policy. En Stiglitz, J., \& Yifu, J. (Edsl.), The Industrial Policy Revolution IThe Role of Government Beyond Ideology (pp. 1-15). New York: Palgrave Macmillan.

Tarr, A. (2008). Los estados como innovadores. En P. Torres \& D. Barceló (Eds.), La reforma del estado. Experiencia mexicana y comparada en las entidades federativas (pp. 55-62.). México: Editorial Porrúa.

Vázquez, A. (2005). Las nuevas fuerzas del desarrollo. Barcelona: Antonio Bosch. Vázquez, M. A. \& Bocanegra, C. (2015). El regionalismo en América del Norte: saldos del modelo TLCAN para México. Revista Aportes para la Integración Latinoamericana, (33), 1-20. doi: 10.24215/24689912e1\%20-\%2020

Vázquez, M. A. \& Hernández, M. C. (2001). Región y grupos económicos en Sonora. Breve itinerario de su integración (1910-1950). Revista Frontera Norte, 13(26), 77-104.

Ventura-Dias, V. (2004). Introduction: Juggling with WTO Rules in Latin America. En Lengyel, M. \& Ventura-Dias, V. (eds.), Trade policy reforms in Latin America: multilateral rules and domestic institutions (pp. 1-23). New York: Palgrave-Macmillan.

Walsh, J. (2016). An Introduction to linking theory. Review of Business \& Finance Studies, 7(2), 91-100. 